\title{
RECURSOS EDUCATIVOS ABIERTOS: REPOSITORIOS Y USO
}

\section{Gema Santos-Hermosa, Núria Ferran-Ferrer y Ernest Abadal}

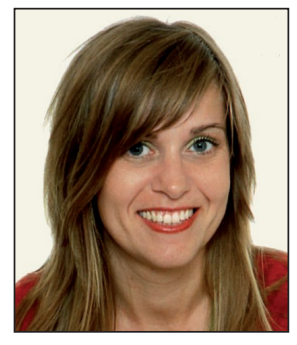

Gema Santos-Hermosa realiza su tesis doctoral sobre "Desarrollo y uso de los recursos educativos abiertos en la docencia universitaria española" en la Universidad de Barcelona (UB). Documentalista en la Biblioteca Virtual de la Universitat Oberta de Catalunya (UOC) y colaboradora docente de los Estudios de Ciencias de la Información y la Comunicación de la misma universidad. Licenciada en historia y en documentación por la UB y master en sociedad de la información y el conocimiento por la UOC.

Universitat Oberta de Catalunya (UOC) Rambla de Poblenou, 156. 08018 Barcelona, España msantoshe@uoc.edu

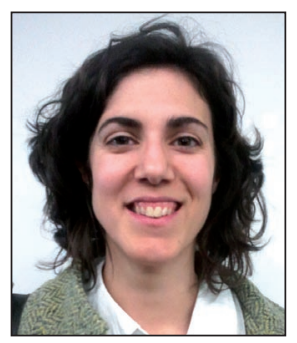

Núria Ferran-Ferrer, profesora de los Estudios de Ciencias de la Información y la Comunicación en la Universitat Oberta de Catalunya (UOC), es adjunta al Vicerrectorado de Investigación e Innovación en la UOC. Doctorada en documentación por la $U B$, licenciada en periodismo por la $U A B$ y en documentación por la UOC. Participa en los proyectos de investigación "Acceso abierto a la ciencia" y "Cultura, ocio y tecnologías digitales".

Universitat Oberta de Catalunya (UOC) Rambla de Poblenou, 156. 08018 Barcelona, España nferranf@uoc.edu

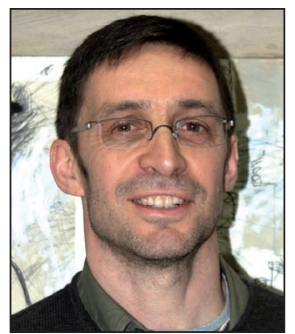

Ernest Abadal es decano y catedrático de la Facultat de Biblioteconomia i Documentació de la Universitat de Barcelona, licenciado en filosofía, diplomado en biblioteconomía y documentación, y doctor en ciencias de la información. Co-director del grupo de investigación "Acceso abierto a la ciencia" (http://accesoabierto.net) y coordinador del grupo de investigación consolidado "Cultura y contenido digitales". Es investigador principal de diversos proyectos del Plan Nacional I+D+l.

Univ. de Barcelona, Fac. de Biblioteconomia i Documentació Melcior de Palau, 140. 08014 Barcelona, España abadal@ub.edu

\section{Resumen}

Se presenta una revisión bibliográfica, estado del arte, historia, características, desarrollo, proyectos y líneas de investigación de los recursos educativos abiertos (OERs), así como su disposición en repositorios y su uso en la práctica educativa universitaria. Los OERs han tenido dos fases: una inicial, preocupada por el acceso, y otra más actual, centrada en su incorporación a la práctica educativa.

\section{Palabras clave}

Acceso abierto, Recursos educativos abiertos, OER, Repositorios institucionales, Práctica educativa, Educación superior.

\section{Title: Open educational resources: repositories and use}

\begin{abstract}
A bibliographic review, state of the art, history, characteristics, development, projects and research lines of open educational resources (OERs) are presented, as well as their storage in repositories and their use in university educational practice. OERs have had two phases: an initial one concerned about providing access, and the more current one, focused on their incorporation into educational practice.
\end{abstract}

\section{Keywords}

Open access, Open educational resources, OER, Institutional repositories, Educational practice, Higher education.

Santos-Hermosa, Gema; Ferran-Ferrer, Núria; Abadal, Ernest. Recursos educativos abiertos: repositorios y uso. El profesional de la información, 2012, marzo-abril, v. 21, n. 2, pp. 136-145. 


\section{Introducción}

El movimiento por el acceso abierto (open access) persigue la difusión libre y gratuita de la producción científica, es decir, los contenidos derivados de la investigación. Esta iniciativa se está ampliando al material docente, a los denominados recursos educativos en abierto (OER), el valor de los cuales se ha visto acrecentado por el Espacio Europeo de Educación Superior (EEES) y, en especial, por un nuevo modelo pedagógico que promueve que docentes y estudiantes usen, reutilicen y compartan recursos con el resto de la comunidad educativa.

Los OERs tienen sus inicios en 2001, cuando el Massachusetts Institute of Technology (MIT) creó el programa OpenCourseWare $(O C W)$ y desde entonces el interés por ellos ha ido aumentando. En estos once años los contenidos abiertos para la educación han seguido dos fases de desarrollo: la inicial, focalizada en proporcionar acceso a los contenidos y la actual, más preocupada por el uso por su incorporación en las prácticas educativas.

\section{Recursos educativos abiertos}

\subsection{Definición y características}

El término "recursos educativos abiertos" -en inglés, open educational resources (OER)- fue acuñado en 2002 por la Unesco, aunque anteriormente ya se utilizaba "contenido de código abierto" (Wiley, 1998), expresión que procede del entorno del software de código abierto. Los OERs son los materiales y recursos educativos que pueden ser reutilizados para la docencia y el aprendizaje de forma gratuita (Unesco, 2002). Las definiciones de los OERs varían en función del aspecto que se quiere enfatizar, ya sea la reutilización o el ámbito de aplicación (Atkins, Brown y Hammond, 2007).

Los OERs son un denominador genérico que incluye cursos y programas curriculares, módulos didácticos, guías de estudiante, libros de texto, artículos de investigación, vídeos, podcasts, herramientas de evaluación, materiales interactivos (como simulaciones), bases de datos, software, aplicaciones (incluyendo aplicaciones móviles) y cualquier otro material educativo diseñado para uso en la enseñanza y el aprendizaje (OECD, 2007). En este sentido, no deben confundirse con los contenidos del proyecto OpenCourseWare (OCW), un conjunto de materiales educativos de alta calidad organizados en cursos que a menudo incluyen una planificación y herramientas de evaluación, y que serían, por tanto, un tipo específico de OER.

Las principales características de los OERs son:

- la accesibilidad, entendida como la disponibilidad del recurso a ser localizado y utilizado en cualquier lugar o momento;

- la reusabilidad, propiedad a ser modificado y utilizado en diferentes contextos de aprendizaje;

- la interoperabilidad, o facilidad de ser adaptado e interconectado entre diferentes hardwares, dispositivos o herramientas;

- la sostenibilidad, funcionamiento correcto a pesar de los cambios de versiones, de software, etc.
- los metadatos, o descripciones que posibilitan su indexación, almacenamiento, búsqueda y recuperación (Varlamis; Apostolakis, 2006; Olcos, 2007).

Hay que matizar que el término OER no es sinónimo de aprendizaje online o e-learning, ni de educación abierta. Si bien es cierto que algunos cursos de e-learning aprovechan OERs, esto no significa que se identifiquen exclusivamente con el aprendizaje online. De hecho, muchos de los recursos abiertos que se producen en la actualidad, aunque se comparten en formato digital, también son imprimibles. Por otro lado, aunque el uso de OERs pueda apoyar la idea de la educación abierta, ésta no sólo implica liberar recursos -y por ende usar OERs en los programas educativos- sino que también requiere otros aspectos que faciliten dicha apertura -como el análisis sistemático de evaluación y sistemas de acreditación, el apoyo a los estudiantes, marcos curriculares, mecanismos para reconocer el aprendizaje previo, etc. (Unesco-IITE, 2011).

\section{El término de recursos educativos abier-} tos (OER) no es sinónimo de aprendizaje online o e-learning, ni de educación abierta ni de OpenCourseWare (OCW)

También en el ámbito del e-learning se han generalizado otros términos como objeto digital educativo (ODE) (Aenor, 2008), learning object (LO) o reusable learning object (RLO). Los LOs, digitales o no, pueden ser usados, reusados o referenciados durante el aprendizaje, la educación o la formación en TIC (IEEE, 2002). Aunque en ocasiones se utilicen como sinónimos de OERs, estos conceptos tienen un fuerte componente tecnológico y están más relacionados con estándares de metadatos educativos (por ejemplo, LOM-ES, versión española del estándar ICEE-LOM para etiquetado de ODEs).

Paradoja de la reutilización: para que el contenido sea usable, debe ser específico en su contexto. Pero si es muy específico entonces es poco reutilizable (Wiley)

\subsection{Antecedentes: evolución, instituciones e iniciati- vas}

Los inicios de los OERs se remontan a 2001 cuando el MIT ofreció en abierto todo el catálogo de sus cursos online, el proyecto $O C W$, provocando un movimiento global de recursos educativos en abierto ${ }^{1}$. La primera manifestación de este fenómeno fue la asociación del MIT con la University of Utah, donde el profesor Wiley creó una red de apoyo compartida para el contenido del OCW distribuido a través de comunidades voluntarias.

En 2005 se creó el OpenCourseWare Consortium, una comunidad mundial formada por instituciones de educación superior y otras organizaciones comprometidas con el avance del OCW y su impacto en la educación. A partir de entonces el número de iniciativas empezó a extenderse rápidamente 
por EUA, Japón y Europa (Wiley, 2006). El impacto del MIT provocó que otras universidades de prestigio internacional se adhirieran, generando proyectos propios en diferentes idiomas: como el OCW-Universia (en castellano y portugués); la CORE's simplified Chinese translation (en chino), o la Chulalongkorn University's Thai translation (en tailandés).

La importancia de los OERs dentro de la comunidad educativa se ha puesto de manifiesto con las numerosas iniciativas llevadas a cabo por la Unesco (2009) y por otras entidades internacionales no gubernamentales para asegurar el derecho universal a la educación y el aprendizaje a lo largo de la vida.

El International Council for Open and Distance Education (ICDE), ha creado el grupo de trabajo OER Taskforce (Mulder y Rikers, 2008) y, más recientemente, la Open Educational Quality Initiative (OPAL) ${ }^{2}$. La OPAL nace con el objetivo de promover la innovación y la mejora de la calidad en la educación usando OERs. Frente al enfoque inicial de promoción del acceso y la reutilización, los proyectos actuales se centran en la innovación y la calidad a través de prácticas educativas abiertas (OPAL, 2011).

El Institute for Information Technologies in Education (IITE), de Unesco, trabaja para la aplicación de las TIC en la educación e implementa modelos de uso de OERs en la Commonwealth of Independent States $(C I S)^{3}$ y otros países de habla no-inglesa (Unesco-IITE, 2011). Bajo la premisa de proporcionar información sobre la diversidad de situaciones en las que se pueden encontrar los OERs, IITE fomenta estudios de caso de diferentes realidades como los de Lituania (Volungevičienė, 2011) y Brasil (Inamorato-Dos-Santos, 2011).

La William and Flora Hewlett Foundation financia desde 2002 proyectos para la mejora de la educación a nivel mundial y ha realizado donaciones para apoyar instituciones que desarrollan o proporcionan OERs. Trabaja para establecer un ecosistema global de OERs flexible y auto-sostenible. Otras líneas de trabajo específicas son las de investigar su impacto en la educación, promover políticas y estrategias de creación de fondos, desarrollar redes para comunidades $\mathrm{K}-12$ (kindergarten to 12, educación primaria y secundaria), etc.

La Open Education Resources Foundation $(O E R F)^{4}$, una organización filantrópica e independiente, proporciona apoyo a educadores e instituciones interesadas en colaborar en proyectos internacionales de aplicación de los OERs en la educación abierta. Su mayor aportación es la creación de comunidades abiertas que trabajan colaborativamente compartiendo conocimiento. Su proyecto estrella, el WikiEducator, consiste en una plataforma global para la creación y reutilización de OERs que facilita la confianza del profesorado con el código abierto.

Entre las iniciativas de la Commonwealth of Learning ( $\mathrm{CoL})$, una organización intergubernamental comprometida en la creación y utilización de OERs, está el desarrollo de una política sobre OERs y el asesoramiento respecto a su potencial para la calidad de la educación superior (Butcher, 2011; CoL y Unesco, 2011).
El fomento de los OERs también se ha convertido en un asunto global de interés para organizaciones gubernamentales. Destacan los programas de creación de repositorios que están llevando a cabo instituciones como el JISC Cetis ${ }^{5}$, en el Reino Unido o el OER Africa ${ }^{6}$, en el continente africano.

Mención aparte merecen las universidades que se han sumado al movimiento de los OERs a través de iniciativas propias e innovadoras, como: la Wiki Library and Wikiversity, dentro del proyecto nacional finlandés AVO (Open Networks for Learning), The 'free' University of the People, la Peer to Peer University (P2PU) y el prototipo OER University (\#oeru).

Finalmente, cabe señalar el interés de la sociedad en general por difundir y compartir OERs a través de comunidades de usuarios (como WikiEducator, Curriki) y plataformas 2.0, como YouTube EDU e iTunes $U$, que permiten acceso instantáneo a vídeos de conferencias, entrevistas en podcast, etc., a la vez que cobran fuerza iniciativas particulares como el canal Teachertrainingvideo ${ }^{7}$.

\subsection{Interoperabilidad}

El desarrollo y aplicación de estándares de accesibilidad y usabilidad de los OERs es un paso determinante para su almacenamiento y posterior interoperabilidad. Se necesitan protocolos y especificaciones de interoperabilidad para hacer compatibles las diversas infraestructuras tecnológicas y para el diseño de contenidos que permitan su descripción, estructuración, etc. (Minguillón; Sicilia; Lamb, 2010). Por este motivo se han ido creando organismos internacionales que regulan estos estándares en el ámbito de las tecnologías educativas y el e-learning (tabla 1 ).

\subsection{Aspectos legales}

La propiedad intelectual es una cuestión clave para los OERs, en especial si tenemos en cuenta que los contenidos están pensados para su reutilización. La aparición de las licencias abiertas se ha visto impulsada por la necesidad de proteger al titular de los derechos de autor, especialmente en entornos web en los que los contenidos pueden ser fácilmente copiados y compartidos (Unesco-IITE, 2011) y también para paliar las limitaciones de "todos los derechos reservados".

Existe un amplio abanico de marcos jurídicos que intentan determinar cómo autorizar el uso de los recursos abiertos. Las licencias más conocidas son las Creative commons (CC), que proporcionan mecanismos legales para garantizar que los autores conserven el reconocimiento de su trabajo (autoría) a la vez que ceden otros derechos autorizando que se pueda compartir, adaptar, restringir o no la actividad comercial de su obra.

\subsection{Calidad}

El proyecto OCW basó la evaluación de los OERs en el prestigio de las instituciones que los habían creado. Este fue el indicador de calidad de los OERs hasta que el número de productores fue tan amplio que hizo impracticable esta medida. Entonces se adoptó el criterio de calidad basado en las revisiones por parte de expertos, de manera similar al funcionamiento de la comunicación científica. De este modo, el colectivo académico que consulta y utiliza los OERs pasa a ser su evaluador. 


\begin{tabular}{|c|c|}
\hline Organismos & Normas y estándares \\
\hline $\begin{array}{l}\text { Instructional Management Systems } \\
\text { Global Learning Consortium }\end{array}$ & $\begin{array}{l}\text { Agrupa centros de investigación, universidades y compañías informáticas que desarrollan especificaciones } \\
\text { para marcar metadatos educativos (IMS LOM), perfiles de estudiantes (IMS Learner Information Package, IMS } \\
\text { LIP), preguntas y exámenes (IMS Question and Test Interoperability, IMS QTI), paquetes (content packaging, IMS } \\
(P) \text { o procesos de enseñanza y aprendizaje (IMS Learning Design, IMS LD) }\end{array}$ \\
\hline $\begin{array}{l}\text { IEEE (Institute of Electrical and } \\
\text { Electronics Engineers), Learning } \\
\text { Technology Standards Committee }\end{array}$ & $\begin{array}{l}\text { Uno de los estándares específicos para metadatos más ampliamente adoptado es LOM (learning object } \\
\text { metadata), que especifica su sintaxis y semántica. Contiene dos elementos relevantes para la descripción de } \\
\text { objetos de aprendizaje: niveles de agregación y tipos de recursos de aprendizaje. }\end{array}$ \\
\hline $\begin{array}{l}\text { ADL (Advanced Distributed } \\
\text { Learning) }\end{array}$ & $\begin{array}{l}\text { A iniciativa del US Dept. of Defense se crea el estándar Scorm (sharable content object reference model), un } \\
\text { conjunto de normas técnicas que permiten crear objetos pedagógicos estructurados y su intercambio } \\
\text { entre diferentes sistemas de gestión de aprendizaje online que se ajusten al mismo estándar. A pesar de } \\
\text { que Scorm es considerado el estándar dominante para contenido digital, tiene limitaciones importantes en } \\
\text { cuanto a la reutilización de recursos educativos y falta de utilidades en el soporte pedagógico (no aprovecha } \\
\text { las capacidades de colaboración y comunicación de la Red). }\end{array}$ \\
\hline IMS Global Learning Consortium & $\begin{array}{l}\text { IMS Learning Design (IMS LD) es una especificación basada en modelos colaborativos de aprendizaje, que } \\
\text { aporta nuevas funcionalidades didácticas. Ofrece un soporte de comunicación para la interacción entre } \\
\text { estudiantes, introduce el papel de profesor y permite combinar recursos educativos con actividades peda- } \\
\text { gógicas y reutilizar estas últimas. }\end{array}$ \\
\hline
\end{tabular}

Tabla 1. Organismos internacionales de estándares aplicados a los OERs [elaborada a partir de Berlanga et al. (2005), EdReNe (2005), Griffiths et al. (2005), y Ververt y Duval (2008)].

Un ejemplo es el proyecto Connexions, formado por una comunidad mundial de autores que trabaja colaborativamente y garantiza la calidad de los OERs contenidos (Baraniuk, 2007).

Otro modelo es el del repositorio Merlot, que cuenta con dos sistemas de revisión: uno informal, que permite a cualquier miembro de la comunidad asignar comentarios y valoraciones, y otro formal, parecido al peer review de cualquier publicación científica (Jones y McNaught, 2010). Ambos presentan problemas ya que el sistema de revisión individual es lento y costoso y el realizado por la comunidad de usuarios tiene una baja participación y adolece de falta de representatividad (Han, 2004; Kay y Knack, 2009).

Un estudio basado en contrastar evaluaciones con encuestas a estudiantes y profesores, concluye que los sistemas existentes no son suficientes para determinar la calidad y los beneficios educativos de los OERs (Akpinar, 2008). Ochoa y Duval (2008) apuestan por medir la calidad de los recursos de manera automática y Sanz-Rodríguez, Dodero-Beardo y Sánchez-Alonso (2010) proponen un indicador automático y único que integre criterios de valoración (opinión de expertos y usuarios), descripción (metadatos) y empíricos (datos de uso).

Recientemente, y en el caso específico de los cursos OCW, ha aparecido un nuevo sello de calidad internacional denominado Epprobate $^{8}$ (Bates, 2012), que consiste en la evaluación desde el punto de vista del e-learning de los contenidos, las especificaciones técnicas y la satisfacción de los usuarios.

\section{Primera fase: acceso a través de repositorios}

Una vez que los OERs han sido creados y disponen de una licencia abierta, la preocupación fundamental consiste en facilitar el acceso a los mismos (OPAL, 2011; D'Antoni, 2006). Los repositorios son la plataforma que asegura un mejor acceso a los OERs (Wiley, 2000), aunque no se puede olvidar que existen otras vías de acceso como los entornos virtuales de aprendizaje (VLE), portales temáticos, comunidades virtuales, wikis, revistas abiertas, redes sociales, etc. (D’Antoni, 2006).
Los repositorios ofrecen varias prestaciones a los OERs: preservación y reutilización de contenido (Akeroyd, 2005; Bueno-de-la-Fuente, 2010), acceso permanente y mayor visibilidad (Gibbons, 2004), y facilidad de la búsqueda y recuperación (Bennett et al., 2008; Unesco, 2009) mediante el uso de metadatos, que los identifican y permiten su intercambio entre diferentes LCMSs (learning content management systems, sistemas de gestión de contenidos de aprendizaje) (Holden, 2003; Unesco, 2009).

\subsection{Tipos y características de los repositorios}

Los repositorios educativos pueden ser fruto de diversas iniciativas (tabla 2). En este artículo nos centraremos en los repositorios institucionales de OERs en el ámbito de la educación superior.

Las universidades se han sumado al movimiento del acceso abierto creando repositorios institucionales para albergar y difundir la producción académica generada por sus miembros en el desarrollo de sus actividades intelectuales. Generalmente no existen repositorios institucionales exclusivamente de recursos educativos sino que acostumbran a ser híbridos, con materiales de investigación y de docencia. Algunas instituciones han creado colecciones específicas para los contenidos de aprendizaje; otras han separado el repositorio educativo del resto de la producción científica. Finalmente hay instituciones que participan en iniciativas OCW, dando acceso a cursos específicos pero sin abarcar el resto de recursos educativos depositados.

Se han podido establecer algunas clasificaciones respecto a los repositorios de OERs. Margaryan et al. (2006) diferencian cuatro grupos por medio de una matriz de dos dimensiones: su cobertura temática o multi-disciplinar y su alcance institucional o inter-institucional (regional, nacional e internacional). Otro estudio (Unesco, 2009) los agrupa según operen a gran o pequeña escala, y por tipo de proveedor (tabla 3).

La mayoría de repositorios incluye documentos de texto, audio, vídeo y gráficos. Los recursos educativos no tienen el objetivo de difundir los resultados de una investigación sino el de ser útiles dentro del proceso de aprendizaje. 


\begin{tabular}{|c|c|c|}
\hline \multicolumn{2}{|c|}{ Tipos de repositorios educativos } & Ejemplos \\
\hline \multirow{5}{*}{$\begin{array}{l}\text { Instituciones propulsoras } \\
\text { o financiadoras }\end{array}$} & Gobiernos & $\begin{array}{l}\text { ERIC (Education Resources Information Center), US Dept of Education } \\
\text { Namcol (Namibian College of Open Learning) }\end{array}$ \\
\hline & Universidades & $\begin{array}{l}\text { OpenLearn, Open University, UK } \\
\text { Open Content, University of Cape Town }\end{array}$ \\
\hline & Instituciones educativas & $\begin{array}{l}\text { OER Commons } \\
\text { Institute for the Study of Knowledge Management in Education (Iskme) }\end{array}$ \\
\hline & Fundaciones & Connexions, financiado por varias fundaciones \\
\hline & Consorcios & $\begin{array}{l}\text { Materials Docents en Xarxa (MDX) y Tesis Doctorals en Xarxa (TDX), Consorci de } \\
\text { Biblioteques Universitàries de Catalunya (CBUC) } \\
\text { CEC LOR, Consortium for Educational Communication, India }\end{array}$ \\
\hline \multirow{2}{*}{$\begin{array}{l}\text { Formato de los } \\
\text { contenidos }\end{array}$} & Audiovisuales & $\begin{array}{l}\text { EUScreen } \\
\text { EduTubePlus }\end{array}$ \\
\hline & Multimedia & Multimedia Teaching Objects \\
\hline \multirow{3}{*}{ Cobertura geo-política } & Regionales & Edu365 y Merlí, Dept. Ensenyament, Generalitat de Catalunya \\
\hline & Estatales & $\begin{array}{l}\text { Federaciones de repositorios con recursos educativos: } \\
\text { Agrega, España } \\
\text { Jorum, UK } \\
\text { NDLR, Irlanda } \\
\text { Merlot, EUA } \\
\text { Lornet, Canadá } \\
\text { NIME, Japón } \\
\text { EdNA Online, Australia }\end{array}$ \\
\hline & Internacionales & $\begin{array}{l}\text { Ariadne } \\
\text { OER Africa } \\
\text { Globe (Global Learning Objects Brokering Exchange) }\end{array}$ \\
\hline \multirow{3}{*}{ Contenido depositado } & OCW (exclusivamente) & $\begin{array}{l}\text { OCW MIT, Nptel, eGyankosh, Doshisha University OCW, Tuffs OCW, OCW National } \\
\text { University of Columbia }\end{array}$ \\
\hline & OERs por temáticas & $\begin{array}{l}\text { Health Digital Repository (salud) } \\
\text { FETP OCW (economía) } \\
\text { AgEcon (agricultura) } \\
\text { BerkleeShares (música) }\end{array}$ \\
\hline & Buscadores de OERs & $\begin{array}{l}\text { OER Commons } \\
\text { Folksemantic } \\
\text { Discover Ed }\end{array}$ \\
\hline
\end{tabular}

Tabla 2. Clasificación y ejemplos de repositorios de OERs [elaborado a partir de OPAL (2011) y Unesco (2011)]

\begin{tabular}{|c|c|c|c|}
\hline \multirow{2}{*}{ 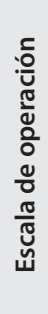 } & $\begin{array}{l}\frac{0}{0} \\
\frac{0}{\pi} \\
\text { ปँ }\end{array}$ & $\begin{array}{l}\text { OpenCourseWare, MIT } \\
\text { OpenLearn, Open University, UK }\end{array}$ & $\begin{array}{l}\text { Wikipedia } \\
\text { Connexions } \\
\text { Merlot } \\
\text { WikiEducator }\end{array}$ \\
\hline & 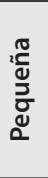 & $\begin{array}{l}\text { OpenER, Open University, Holanda } \\
\text { Free Courseware Project, University of the } \\
\text { Western Cape, Sudáfrica } \\
\text { Klagenfurt OCW, United Nations } \\
\text { University }\end{array}$ & $\begin{array}{l}\text { OpenCourse } \\
\text { Free Curricula } \\
\text { Center } \\
\text { LeMill }\end{array}$ \\
\hline & & Institución & Comunidad \\
\hline & & \multicolumn{2}{|l|}{ Tipo de proveedor } \\
\hline
\end{tabular}

Tabla 3. Categorías de suministradores de OERs (Unesco, 2009)

Aspectos como la granularidad (especificidad o detalle), reutilización e intercambio de los recursos educativos son clave para el éxito de los repositorios (Curda y Kelly, 2009; Bennet et al., 2008), puesto que posibilitan interrelacionar contenidos, actividades y usuarios dentro del contexto de educación abierta (McNaught, 2007). Finalmente, numerosos estudios coinciden en señalar que los repositorios institucionales de OERs pueden aportar beneficios tanto para los docentes y estudiantes como para la propia institución y la sociedad en general (Ferran et al., 2007; Olcos, 2007; Unesco; CoL, 2011; OPAL, 2011; Royster, 2011).

\subsection{Evolución y situación actual de los repositorios}

A nivel mundial se contabiliza un total de 33 repositorios específicamente dedicados a recursos educativos abiertos (ROAR, 2012), y además se podría encontrar este tipo de contenido en un $15 \%$ del total de repositorios (según Open$D O A R, 2012)$.

En Europa el fenómeno del acceso abierto a los recursos educativos es relativamente reciente. Los primeros repositorios de OER datan de 2005 y desde entonces han experimentado un crecimiento lento pero constante. La aparición de Open eLearning Content Observatory Services (Olcos), dentro del programa de e-learning de la Unión Europea, es un ejemplo de la promoción y uso de repositorios educativos. Un estudio sobre el estado del arte de los repositorios de OER en Europa (EdReNe, 2009), señala que aunque la mayoría de los recursos depositados corresponden a la enseñanza primaria y secundaria, cada vez son más importantes los de nivel universitario. Otro estudio más reciente (Roylster, 2011) recoge que el $30,8 \%$ de los repositorios europeos ${ }^{9}$ contiene $^{2}$ cursos, OCW y OERs.

En España las universidades son las instituciones que más se han implicado en el desarrollo y apoyo de los repositorios, 
puesto que el $50 \%{ }^{10}$ dispone de un depósito institucional (Abadal et al., 2011). Aunque la mayor parte de los documentos depositados son de investigación (Melero et al., 2009), durante los últimos años se aprecia un interés y un crecimiento constante del volumen de recursos educativos ${ }^{11}$ (Bueno-de-la-Fuente, 2011). Desde 2006 son 29 las universidades españolas que se han sumado al proyecto $O C W$, un $36 \%$ del total de centros (Abadal et al., 2011).

Destaca el OCW de la Universidad de Alicante, galardonado por el OCW Consortium con un Premio de Excelencia; y el repositorio 02 , la Oberta en Abierto, de la Universitat Oberta de Catalunya (UOC), que se sitúa en los primeros puestos del ranking de directorios y estimador de políticas de acceso abierto Melibea.

\subsection{Comportamiento de los usuarios de los reposi- torios}

Se observan actitudes positivas de los docentes respecto a la utilidad de los repositorios, a la participación en los mismos (Hardin, 2010) y por preservar, reutilizar y crear nuevos OERs (Gadd, 2008). Mejorar la docencia, fomentar la motivación de los estudiantes y crear un vínculo con la institución son algunos de los motivos referidos para contribuir en los repositorios (Bates, 2007), además de razones más altruistas relacionadas con los movimientos Open access (OA) y Free and open source software (FOSS) (Fitzgerald et al., 2006).

Por otro lado, existe cierta unanimidad en cuanto a las reticencias manifestadas para la no contribución: barreras técnicas, carencia de incentivos y desconocimiento de los repositorios (Bates, 2007), a lo que se añade la falta de familiaridad, la inercia y la poca facilidad de uso (Caris, 2004) y la incapacidad de encontrar y reutilizar los recursos depositados (Harley, 2008; Carson, 2010). Koppi y Lavitt (2004) concluyen que una de las acciones a llevar a cabo para incentivar la producción y uso de OERs en la práctica educativa es que las instituciones establezcan un sistema de reconocimiento académico.

\section{Segunda fase: uso en la práctica educacional}

En una segunda fase de desarrollo de los OERs se observa un cambio de enfoque, ya que pasamos del énfasis en el acceso al uso e integración en las prácticas educativas. La aplicación del ciclo de vida de la tecnología (Rogers, 1983) sugiere que los OERs han pasado por una fase de innovación, están llegando a su aceptación y aspiran a llegar a la primera mayoría. Un análisis de las iniciativas de OER a nivel mundial (Stacey, 2010) muestra el predominio de la creación y publicación de recursos educativos pero también apunta a que todavía no se ha llegado a unos niveles de uso y reutilización adecuados. Es por ello que los últimos estudios se centran en analizar el uso de los OERs, evaluando la percepción y prácticas de los usuarios y abriendo vías de integración docente (OPAL, 2011).

\subsection{Uso y percepciones}

La utilización de los OERs también tiene detractores entre la comunidad académica. Algunos profesores creen que no son aptos para sus disciplinas, manifiestan dudas sobre el efecto de su reutilización en la calidad de los contenidos, y además están preocupados por el reconocimiento de las autorías (Lee et al., 2008). Los editores también presentan objeciones basadas en la amenaza que los OERs pueden suponer para su negocio, en tanto que recursos con licencia abierta.

Por su parte las instituciones universitarias, ante los recortes presupuestarios, apuestan por los recursos abiertos. En este contexto, los OERs proporcionan una vía posible a la sostenibilidad financiera de las instituciones universitarias (Olcos, 2007; Friesen, 2009; García y López, 2011).

\subsection{Integración educacional}

La concepción del aprendizaje como "conversación" (Sharples, 2005) permite concebir los OERs desde otra óptica: no como objetos estáticos sino como un flujo dinámico que facilita la redifusión del aprendizaje. Siguiendo a Downes (2008), los OERs podrían considerarse como "palabras" de un vocabulario utilizado por estudiantes y profesores en una "conversación" permanente dentro de una disciplina temática. Se está evolucionando de una cultura de intercambio a una de participación (Atkins, Brown y Hammond, 2007; $O E C D, 2007)$. La filosofía del acceso abierto y el entorno colaborativo facilitan la mejora de la calidad tanto en la docencia como en el aprendizaje (Plotkin, 2010; Downes, 2011; Casserly y Smith, 2008). Los OERs permiten el acceso al aprendizaje a lo largo de la vida a todo el mundo.

La educación es una relación de intercambio en la que la actitud abierta es imprescindible. Cuanto más abiertos seamos, mejor será la educación (Wiley)

Finalmente, cabe destacar que los OERs se han convertido en una herramienta de apoyo a la transformación educativa. Casserly y Smith (2008) señalan que tienen un potencial, aún no probado, de transformación de la enseñanza y el aprendizaje. Mientras que su valor educativo radica en utilizar los recursos como un método integral de comunicación dentro de los planes de estudio, su poder transformador reside en la facilidad con que tales recursos pueden compartirse a través de internet (Bucher, 2011). La directora ejecutiva del OCW Consortium, Mary-Lou Forward, afirma que la razón por la que las instituciones y sus docentes promueven y utilizan OERs es porque creen que el conocimiento compartido es la base del conocimiento.

Desde una perspectiva constructivista, el conocimiento no debe transmitirse del profesor al alumno sino que el primero debe asumir el rol de facilitador del aprendizaje y enfatizar las actividades de los estudiantes con las que desarrollen competencias, conocimientos y habilidades. El éxito de los OERs sólo será posible si hay un cambio en la cultura en el sector educativo y los profesores y estudiantes se ven como productores y adaptadores de contenido en vez de simples consumidores (Olcos, 2007).

\section{Conclusiones}

A partir de la revisión bibliográfica realizada, hemos constatado que los OERs son una área de investigación emergente, 
que ha sido tratada desde perspectivas variadas. Las características, usos y aplicaciones de los OERs no son estáticas sino que están evolucionando y no se han desarrollado en toda su magnitud. La garantía de calidad y la acreditación de los OERs tampoco han llegado a su máximo apogeo y sólo se incrementará su importancia cuando el movimiento OA se afiance y el volumen de contenidos y el número de usuarios aumente. Los repositorios de OERs son un campo de estudio aún en fase de consolidación, como también lo es la integración de sus contenidos en las prácticas de aprendizaje.

Entre los elementos esenciales de los OERs que se están estudiando en estos momentos, podemos destacar la reutilización de los materiales, la creación de contenidos por parte de los autores, la implementación de prestaciones para la valoración social, o su uso en el ámbito universitario. Se trata de elementos que van a condicionar el desarrollo futuro de los OERs y sobre los que vamos a realizar un breve comentario.

Como se ha indicado, una de las particularidades más destacadas de los OERs es que puedan ser remezclados y redefinidos para formar nuevos recursos de contenido que funcionan de manera autónoma, conectados e interactuando, dentro de los entornos de aprendizaje. En los últimos años ha aparecido un nuevo modelo de educación donde la docencia y el aprendizaje se realizan en comunidad, de forma más autónoma, por estudiantes guiados y asistidos por educadores y expertos.

El panorama de la edición de contenidos educativos está cambiando y la exclusividad que tenía la industria editorial empieza a disminuir frente al desarrollo autónomo de contenidos por parte de los propios autores. El fenómeno social de la producción de código abierto se ha extendido recientemente a los OERs, provocando una descentralización en la creación y reutilización de los mismos entre profesores y estudiantes. El software libre apoya la edición colaborativa de recursos educativos abiertos y, en este sentido, amplia su concepto de apoyo a los contenidos educativos para identificarse también con el proceso de aprendizaje.

Los repositorios de OERs están empezando a aplicar dinámicas sociales con el objetivo de que los usuarios publiquen comentarios sobre los recursos que han consultado, los marquen y los envíen por correo electrónico, etc.

A pesar de que los OERs son, cada vez más, una prioridad en la agenda de las políticas educativas sociales y reciben el apoyo de muchos actores del ámbito educativo, su uso en la educación superior todavía no ha alcanzado el umbral crítico para ofrecer un aprendizaje de calidad y una formación continuada a los ciudadanos.

De acuerdo con los temas apuntados en esta revisión, una tendencia interesante de investigación futura será estudiar el papel de los OERs y las arquitecturas de aprendizaje abiertas dentro del EEES que transformarán la educación del siglo XXI.

\section{Notas}

1. En 2002 el MIT estrenó el primer OCW con 50 cursos.

2. Organización financiada por la Comisión Europea de Edu- cación y Formación, que nació gracias a organizaciones internacionales como la Unesco, ICDE y Efquel.

3. Países resultantes de la disolución de la Unión Soviética en 1991

4. Algunos de los socios fundadores son el Otago Polytechnic (Nueva Zelanda), la Southern Queensland University (Australia) y la Athabasca University (Canadá).

5. El Centre for Educational Technology and Interoperability Standards (Cetis) trabaja en el fomento de los OERs conjuntamente con el Joint Information Systems Committee (JISC) y la Higher Education Academy (UK).

6. Iniciativa del South African Institute for Distance Education (Saide).

\section{7. http://www.teachertrainingvideos.com}

8. A iniciativa de la Learning Agency Network (Laneto), la Agence Wallonne des Télécommunications (AWT) y el eLearning Quality Service Center (eLQSC)

9. Frente al $14,3 \%$ de EUA y el $10 \%$ canadiense. Un $72,3 \%$ del total de repositorios participantes corresponden al ámbito universitario.

10. Este porcentaje equivale a 37 universidades y 48 repositorios; que añadiendo los 5 repositorios consorciados del $C B \cup C$, suman un total de 53 repositorios universitarios (2012).

\section{OpenDOAR contabiliza 12 repositorios de OERs (2012).}

\section{Agradecimientos}

Este estudio se ha realizado en el marco del proyecto "El acceso abierto a la producción científica (open access) en España: análisis del grado de implantación y de la sostenibilidad de un nuevo modelo de comunicación científica", financiado por el Plan Nacional de I+D (CSO2011-29503-C0201/SOCI).

\section{Bibliografía}

Abadal, Ernest; Ollé, Candela; Abad-García, Francisca; Melero, Remedios. "El acceso abierto a la ciencia en las universidades españolas". Revista española de documentación científica [en prensa].

Aenor. "GT9/GT8-SC36/Aenor. Perfil de aplicación LOM-ES1 V.1.0", 2008.

Akeroyd, John. "Information management and e-learning. Some perspectives". Aslib procs: New information perspectives, 2005, v. 57, n. 2, pp. 157-167.

http://dx.doi.org/10.1108/00012530510589128

Akpinar, Yavuz. "Validation of a learning object review instrument: relationship between ratingsof learning objects and actual learning outcomes". Interdisciplinary journal of knowledge and learning objects, 2008, v. 4, pp. 291-302. http://www.ijello.org/Volume4/IJELLOv4p291-302Akpinar. $p d f$

Atkins, Daniel; Brown, John; Hammond, Allen. "A review of the openeducational resources (OER) movement: achie- 
vements, challenges, and new opportunities", 2007. Menlo Park, CA: The William and Flora Hewlett Foundation.

http://www.hewlett.org/uploads/files/Hewlett_OER_ report.pdf

Baraniuk, Richard. Challenges and opportunities for the open education movement: a Connexions case study. Connexions and Rice University, 2007.

http://citadel.cnx.rice.edu:8180/risa/docs/presskit/cnxbro churesposter/baraniuk-MIT-press-chapter-oct07.pdf

Bates, Melanie et al. "Attitudes to the rights and rewards for author contributions to repositories for teaching and learning". ALT-J Research in learning technology, 2007, v. 15, n. 1, pp. 67-82.

http://repository.alt.ac.uk/678/1/ALT_J_Vol15_No1_2007_ Attitudes_to_the_rights_and_re.pdf

Bates, Tony. "New international quality label for e-learning courseware". Online learning and distance education resources, n. 7, 2012.

http://www.tonybates.ca/2012/01/07/new-internationalquality-label-for-e-learning-courseware

Bennett, Sue et al. "A need analysis framework for the design of digital repositories in higher education". En: Lockyer [et al.] (eds.). Handbook of research on learning design and learning objects: issues, applications and technologies. Information Science Publishing, 2008.

Berlanga, Adriana et al. "IMS learning: hacia la descripción estandarizada de los procesos de enseñanza". En: VI Congreso nacional de informática educativa, Simposio nacional de tecnologías de la información y las comunicaciones en la educación (Sintice) 2005, p. 95-102.

Bueno-de-la-Fuente, Gema. Modelo de repositorio institucional de contenido educativo (RICE): la gestión de materiales digitales de docencia y aprendizaje en la biblioteca universitaria [tesis doctoral]. Universidad Carlos III de Madrid, 2010.

http://e-archivo.uc3m.es/handle/10016/9154

Bueno-de-la-Fuente, Gema; Hernández-Pérez, Tony. "Estrategias para el éxito de los repositorios institucionales de contenido educativo en las bibliotecas digitales universitarias". BiD: textos universitaris de biblioteconomia i documentació, 2011, junio, n. 26.

http://www.ub.edu/bid/26/bueno2.htm

Butcher, Neil. A basic guide to OER. British Columbia/Paris: CoL \& Unesco, 2001.

http://www.col.org/resources/publications/Pages/detail. aspx?PID=357

Caris, Mieke. "Why don't faculty use learning object repositories?". En: Cantoni \& C. McLoughlin (Eds.), Procs. of World conf. on educational multimedia, hypermedia and telecommunications. Chesapeake, VA: AACE, 2004 pp. 2838-2840.

Carson, Stephen. Why bother being open?. 22 Nov. 2010. http://opencontent.org/blog/archives/1735

Casserly, Catherine; Smith, Marshall. "Revolutionizing education through innovation: can openness transform teaching and learning?". En: Opening up education: the collective advancement of education through open technology, open content, and open knowledge. Toru liyoshi and M. S. Vijay Kumar (ed). The MIT Press, 2008.

CoL; Unesco. Guidelines for OER in higher education. CoL, 2011.

http://www.col.org/resources/publications/Pages/detail. aspx?PID=364

Cueva, Samanta; Rodríguez, Germania. "OER, estándares y tendencias". Revista de universidad y sociedad del conocimiento (RUSC), 2010, v. 7, n. 1. UOC.

http://rusc.uoc.edu/ojs/index.php/rusc/article/view/v7n1 cueva_rodriguez/v7n1_cueva_rodriguez

Curda, Leslie; Kelly, Melissa. "Guidelines for developing learning object repositories". En: Lockyer L. [et al.] (eds.). Handbook of research on learning design and learning objects: issues, applications and technologies. Information Science Publishing, 2008, pp. 744-760.

D’Antoni, Susan. "The Virtual University, models and messages. Lessons from case studies". The virtual university and e-learning. Unesco-IIEP, 2006.

http://www.unesco.org/iiep/virtualuniversity/home.php

D’Antoni, Susan; Savage, Catriona (ed.). Open Educational Resources. Conversations in cyberspace. Paris: Unesco, 2009.

Downes, Stephen. The future of online learning: ten years on, 2008.

http://www.downes.ca/files/future2008.doc

Downes, Stephen. Open educational resources: a definition, 2011.

http://halfanhour.blogspot.com/2011/07/open-educatio nal-resources-definition.html

EdReNe. State of the art II: educational repositories in Europe, 2009.

http://edrene.org/results/deliverables/EdReNeD3.4TSR_ Repository_strategies.pdf

Ferran, Núria et al. "Enriching e-learning metadata through digital library usage analysis". The electronic library, 2007, v. 25, n. 2, pp. 148-165.

http://dx.doi.org/10.1108/02640470710741296

Fitzgerald, Brian et al. Creating a legal framework for copyright management of open access within the Australian academic and research sector. Brisbane, Queensland University of Technology, 2006. ISBN: 978098029881

http://eprints.qut.edu.au/6099/1/Printed_Oak_Law_ Project Report.pdf

Friesen, Norm. "Open educational resources: new possibilities for change and sustainability". The int review of research in open and distance learning, 2009, v. 10, n. 5, 13 pp. http://www.irrodl.org/index.php/irrodl/article/view/664/1391

Gadd, Elisabeth. "A comparison of academics' attitudes towards the rights protection of their research and teaching materials". Active learning in higher education, 2008, v. 9, pp. $103-121$.

García, Iolanda; López, Cristina. "Los recursos de aprendizaje”. En: Gros, Begoña (ed.). Evolución y retos de la educación virtual. Construyendo el e-learning del siglo XXI. Editorial UOC, 2011, pp. 93-144. 
http://openaccess.uoc.edu/webapps/o2/bitstream/10609/ 9781/1/TRIPA_e-learning_castellano.pdf

Gibbons, Susan. "Benefits of an institutional repository". $\mathrm{Li}-$ brary technology reports, 2004, v. 40, n. 4, pp. 11-16.

Griffiths, David et al. "Learning design tools". En: Koper \& Tattersall (Eds.). Learning design: modelling and implementing network-based education \& training. Springer Verlag, 2005, pp. 109-135.

Han, Kate. Quality rating of learning objects using Bayesian belief networks. PhD thesis. Canada: Simon Fraser University, 2004.

Hardin, Joseph; Cañero, Aristóteles. "Faculty and student perspectives toward open courseware, and open access publishing: some comparisons between European and North American populations". En: Open ED 2010 procs. Barcelona: UOC, 2010.

http://hdl.handle.net/10609/5261

Harley, Diane. "Why understanding the use and users of OERs matters". En: Opening up education the collective advancement of education through open technology open content and open knowledge. The MIT Press, 2008, pp. 197211.

Holden, Colin. "Learning repositories and the Global learning repositories summit". Academic ADL Co-Lab, 2003.

http://academiccolab.org/resources/FinalSummitReport. $p d f$

IEEE. "Draft standard for learning object metadata". New York: Institute of Electrical and Electronics Engineers, 2002. http://Itsc.ieee.org/wg12/files/LOM_1484_12_1_v1_Final_ Draft.pdf

Inamorato-Dos-Santos, Andreia. "Open educational resources in Brazil: state-of-the-art, challenges and prospects for development and innovation". Unesco IITE, 2011.

http://iite.unesco.org/pics/publications/en/files/3214695. $p d f$

IIEP. "Discussion on free and open source software (FOSS) for open educational resources". Discussion log, week 2, 2327 Oct. 2006.

http://oerwiki.iiep.unesco.org/images/6/6f/FOSS_for OER_log_wk2.pdf

Jones, Jennifer; McNaught, Carmel. "Learning object evaluation and reuse". En: Ferran, N; Minguillón, J. (eds.) Content management for e-learning, Nueva York: Springer, 2010.

Lee, Mary et al. "Expanding the reach of health sciences education and empowering others: the OpenCourseWare initiative at Tufts University". Medical teacher, 2008, v. 30, n. 2, pp. 159-163.

http://hdl.handle.net/10427/48160

Kay, Robin; Knaack, Liesel. “Assessing learning, quality and engagement in learning objects: the Learning object evaluation scale for students (Loes-s)". Educational technology research and development, 2009, v. 57, n. 2, pp. 147-168. http://dx.doi.org/10.1007/s11423-008-9094-5

Koppi, Tony; Bogle, Lisa; Lavitt, Neil. "Institutional use of learning objects: lessons learned and future directions". Jo- urnal of educational multimedia and hypermedia, 2004, v. 13, n. 4, pp. 449-463.

Margarya, Anoush. "CD-LOR deliverable 1: report on learning communities and repositories". Glasgow: Centre for Academic Practice and Learning Enhancement, 2006.

http://academy.gcal.ac.uk/cd-lor/learningcommunitiesre port.pdf

Masson, Patrick; Udas, Ken. "An agile approach to managing open educational resources". Connexions, 2009. http://cnx.org/content/m32419/1.1

McNaught, Carmel. "Developing criteria for successful learning repositories". En: Filipe et al. (eds.). Web information systems and technologies. Int confs. Berlin; Heidelberg: Springer, v. 1, pp. 8-18.

Melero, Remedios et al. "The situation of open access institutional repositories in Spain: 2009 report". Information research, 2009, v. 14, n. 4

http://InformationR.net/ir/14-4/paper415.htm/

Minguillón, Julià; Sicilia, Miguel-Ángel; Lamb, Brian. "From content management to e-learning content repositories". En: Ferran, N; Minguillón, J. (eds.) Content management for e-learning. Nueva York: Springer, 2010.

Mulder, Fred; Rikers, Jos. "Open educational resources and open, flexible and distance learning". Final report, ICDE Task Force on Open Educational Resources, 2008.

http://www.icde.org/Final+Report+from+the+OER+Task+Fo rce.9UFRzG4_.ips

Ochoa, Xavier; Duval, Erick. "Relevance ranking metrics for learning objects". IEEE Transactions on learning technologies, 2008, v. 1, n. 1, pp. 34-48.

http://dx.doi.org/10.1109/TLT.2008.1

OECD. Giving knowledge for free: the emergence of open educational resources. Paris, 2007.

Olcos. Educational practices and resources. Olcos Roadmap 2012. Geser (ed.), 2007.

http://www.olcos.org/cms/upload/docs/olcos_roadmap_ summary.pdf

OPAL. "Beyond OER: Shifting focus from resources to practices". The OPAL report 2011.

http://duepublico.uni-duisburg-essen.de/servlets/DerivateServlet/Derivate-25907/OPALReport2011-Beyond-OER.pdf

OpenDOAR. The Directory of Open Access Repositories, 2012.

http://www.opendoar.org

Plotkin, Hal. Free to learn. An open educational resources policy development guidebook for community college governance officials, 2010.

http://wiki.creativecommons.org/images/6/67/Freeto LearnGuide.pdf

ROAR. Registry of Open Access Repositories, 2012. http://roar.eprints.org

Rogers, Everett. "Diffusion of innovations". The Free Press, New York, 1983.

Royster, Paul. "Review of the survey of institutional digital 
repositories, 2011 edition by Primary Research Group", Faculty Publications, UNL Libraries. Paper 223, 2011.

http://digitalcommons.unl.edu/libraryscience/22

Sanz-Rodríguez, Javier; Dodero-Beardo, Juan-Manuel; Sánchez-Alonso, Salvador. "Determinando la relevancia de los recursos educativos abiertos a través de la integración de diferentes indicadores de calidad". Revista de universidad y sociedad del conocimiento (RUSC), 2010, v. 8, n. 2, pp. 46-60. http://rusc.uoc.edu/ojs/index.php/rusc/article/view/v8n2sanz-dodero-sanchez/v8n2-sanz-doderosanchez

Sharples, Mike. "Learning as conversation: transforming education in the mobile age". Conf on seeing, understanding, learning in the mobile age, Budapest, Hungary, 2005. http://www.fil.hu/mobil/2005/Sharples_final.pdf

Stacey, Paul. "Foundation funded OER vs. tax payer funded OER - A tale of two mandates". En: Open ED 2010 proceedings. Barcelona: UOC.

http://hdl.handle.net/10609/5241

Unesco. Forum on the impact of open courseware for higher education developing countries. Final report. Paris, 2002. http://www.wcet.info/resources/publications/unescofinal report.pdf

Unesco. World conf. on higher education: the new dynamics of higher education and research for societal change and development. Paris, 5-8 July 2009.

http://www.unesco.org/fileadmin/MULTIMEDIA/HQ/ED/ ED/pdf/WCHE_2009/FINAL\%20COMMUNIQUE\%20WCHE\% 202009.pdf

Unesco-IIEP. Open educational resources open content for higher education. Final forum report. 24 Oct.-2 Dec. 2005. http://www.unesco.org/iiep/virtualuniversity/media/ forum/oer_forum_final_report.pdf

Unesco-IIEP. Open educational resources open content for higher education. Discussion on free and open source software (FOSS) for open educational resources. 23-27 Oct. 2006.

http://oerwiki.iiep.unesco.org/images/6/6f/FOSS_for_ OER_log_wk2.pdf

Unesco-IITE. "ICT in teacher education: policy, open educational resources and partnership". Procs of int conf IITE2010. Unesco, 2011.

http://unesdoc.unesco.org/images/0019/001936/193658e. $p d f$

Verbert, Katrien; Duval, Erick. "Alocom: a generic content model for learning objects". Intl journal on digital libraries, 2008 , v. 9 , n. 1 , pp. 41-63.

http://dx.doi.org/10.1007/s00799-008-0039-8

Volungevičienè, Airina; Teresevičienè, Margarita. “Open educational resources in Lithuania: state-of-the-art, challenges and prospects for development". Unesco IITE, 2011. http://unesdoc.unesco.org/images/0021/002144/214493e. $p d f$

Wiley, David. "Open content". Opencontent.org, 1998.

Wiley, David. "Connecting learning objects to instructional design theory: a definition, a metaphor, and a taxonomy". En: D.A. Wiley (Ed.). The instructional use of learning objects: online version, 2000.

http://reusability.org/read

Wiley, David. "On the sustainability of open educational resource initiatives in higher education". OECD, 2006. http://www.oecd.org/dataoecd/33/9/38645447.pdf

\section{Próximos temas centrales}

Mayo 2012

Julio 2012

Septiembre 2012

Noviembre 2012

Enero 2013

Marzo 2013

Mayo 2013

Julio 2013
Organización del conocimiento

Comunicación digital

Información y derecho

Bibliotecas académicas

Soportes digitales

Educación y biblioteca

Bibliotecas y documentación de museos

Gestión de contenidos

Los interesados pueden remitir notas, artículos, propuestas, publicidad, comentarios, etc., sobre estos temas a: http://recyt.fecyt.es/index.php/EPI/index 